\title{
Brain-immune interactions in health and disease
}

\author{
Adam Denes ${ }^{1,2 *}$ and Jaleel A. Miyan ${ }^{1}$ \\ ${ }^{1}$ Faculty of Life Sciences, University of Manchester, Manchester, UK \\ ${ }^{2}$ Laboratory of Molecular Neuroendocrinology, Institute of Experimental Medicine, Budapest, Hungary \\ *Correspondence: adam.denes@manchester.ac.uk
}

Edited and reviewed by:

Hubert Vaudry, University of Rouen, France

Keywords: brain-immune interactions, neuroinflammation, systemic responses, bi-directional communication, disease

Modern medicine cannot avoid the understanding of the fine-tuned communication between the many, seemingly distinct systems in the body. An excellent example of this is the challenge to understand the bi-directional communication between the brain and the immune system. Brain-immune interactions take place in different organs, involving a wide range of cells and mediators, coordinated through sensory and effector pathways in the central nervous system (Ader et al., 1990; Elenkov et al., 2000; Rivest, 2009). The interactions work in both directions to maintain a healthy state of body and brain in the face of diverse, harmful challenges from foodstuffs, toxins, allergens, infective agents, or injury. Dysfunction and inappropriate regulation of inflammatory or neuronal responses underlie many diseases that have become more prevalent in recent decades, predominantly in developed countries. These countries are also characterized by an increased aging population and profoundly increased cost to healthcare due to age-related brain conditions including dementia and other neurodegenerative diseases. Recent research has established a significant role for the immune system in several brain diseases including multiple sclerosis, tumors, stroke, mental disorders, Alzeimer's, and Parkinson's disease. In turn, mood disorders, stress, autonomic dysfunction, acute, and chronic brain injury have been linked with the development of organ failure, cancer, heart disease, systemic inflammatory conditions, infections, and hematological diseases further implicating dependent interrelationships between the immune system and the brain (Denes et al., 2010; Moreno-Smith et al., 2010; Deretzi et al., 2011; Iadecola and Anrather, 2011; Wraith and Nicholson, 2012; Theoharides et al., 2013; Heneka et al., 2014). Both preclinical and clinical research have contributed significantly to our knowledge about these interactions, yet another major challenge is to translate multiple research findings into clinical benefit.

The papers in this research topic discuss some of the most pressing issues concerning the interactions between the neural and immune systems. Murakami and colleagues present their research findings and their "gateway" theory of how regional neuronal responses can drive the migration of autoreactive $\mathrm{T}$ cells across the cerebrovascular endothelium to particular sites of the brain where they contribute to the development of experimental autoimmune encephalomyelitis (EAE), a mouse model of multiple sclerosis (Kamimura et al., 2013). They also show that regional neural stimulation can therapeutically prevent the gating through blood vessels. Geenen et al. discuss how autoimmunity directed against neuroendocrine glands could be due to genetic or acquired problems that affect the presentation of neuroendocrine self-peptides in the thymus (Geenen et al., 2013). This process in the thymus is normally responsible for the clonal deletion of self-reactive $\mathrm{T}$ cells and the generation of regulatory $\mathrm{T}$ cells. Their findings could thus support the development of novel treatment strategies against type 1 diabetes for example.

In their comprehensive review article, Anrather and colleagues describe how reprogramming of local and systemic immune mechanisms contributes to the induction of cerebral ischemic tolerance, a process that is characterized by protection against the ischemic injury after application of ischemic stress to one tissue or organ (Garcia-Bonilla et al., 2014). Appropriate reprogramming of key immune mechanisms could be used to develop novel stroke therapies including possible prevention of injury through stroke in vulnerable individuals. The research paper by Denes et al. demonstrates that brain injury, anesthesia, and surgical interventions have diverse systemic consequences, including altered leukocyte responses in several organs of the body and rapid mobilization of granulocytes (Denes et al., 2013). This could have important implications for animal models of cerebral ischemia as well as for patients with brain injury or for those undergoing surgeries or exposed to prolonged anesthesia. The review article by Möller and his colleagues focuses on the regulation of the kynurenine pathway by inflammatory mediators and how this contributes to neurodegenerative and psychiatric disorders (Campbell et al., 2014). They also highlight the potential for therapeutic interventions by modulation of the kynurenine pathway.

Assas and colleagues discuss important aspects of neuroimmune communication and show how sensory fibers containing the neuropeptide calcitonin gene-related peptide (CGRP) shape the responses of macrophages, mast cells and other immune cells throughout the body and how these interactions contribute to immune defense and diverse inflammatory conditions (Assas et al., 2014). This neuropeptide and the c class nerve fibers that contain it thus form a key pathway for bi-directional neuroimmune interactions and could form a target for future neuroimmune based therapies.

Neuro-immune abnormalities not only affect adults and the elderly, but also play a role in diverse diseases that manifest in children. D'Angiulli et al. show that children in the Mexico City Metropolitan Area, who are chronically exposed to high concentrations of air pollutants, present with increased amounts of inflammatory mediators along with accumulation of misfolded 
proteins in the cerebrospinal fluid (Calderon-Garciduenas et al., 2013). They propose that environmental factors could mediate detrimental actions in the developing brain.

Paul Ashwood and colleagues report that the behavioral characteristics, including social deficits, repetitive grooming behavior and atypical vocalizations, observed in BTBR $\mathrm{T}+\mathrm{tf} / \mathrm{J}$ mice are associated with the development of an inflammatory macrophage phenotype in this strain (Onore et al., 2013). They suggest that such a relationship between elevated inflammatory burden and repetitive grooming behavior may have relevance to the repetitive and stereotyped behavior characteristic of autism since many Autistic children also present with an increased inflammatory profile. Goyal and Miyan review the possible role of neuroimmune abnormalities in autism (Goyal and Miyan, 2014). They highlight the influence of environmental factors on the abnormal neurological, immunological, and neuroimmunological functions reported in Autistic children and discuss how these interactions can lead to or exacerbate autism spectrum disorder. Their discussion links poor development of the neuroimmune system to vulnerability to these environmental challenges and the consequential effects on the brain and its functions.

We hope that the articles presented in this research topic give thought-provoking and valuable insight into some of the important aspects of brain-immune interactions. Neuro-immune processes are likely to contribute to diverse pathologies in both the periphery and the brain leading to complex human diseases that affect millions of people worldwide. Understanding mechanisms of neuro-immune interactions could help to find appropriate therapies to some of these conditions.

\section{REFERENCES}

Ader, R., Felten, D., and Cohen, N. (1990). Interactions between the brain and the immune system. Annu. Rev. Pharmacol. Toxicol. 30, 561-602. doi: 10.1146/annurev.pa.30.040190.003021

Assas, B. M., Pennock, J. I., and Miyan, J. A. (2014). Calcitonin gene-related peptide is a key neurotransmitter in the neuro-immune axis. Front. Neurosci. 8:23. doi: $10.3389 /$ fnins.2014.00023

Calderon-Garciduenas, L., Cross, J. V., Franco-Lira, M., Aragon-Flores, M., Kavanaugh, M., Torres-Jardon, R., et al. (2013). Brain immune interactions and air pollution: macrophage inhibitory factor (MIF), prion cellular protein $(\operatorname{PrP}(\mathrm{C})$ ), Interleukin-6 (IL-6), interleukin 1 receptor antagonist (IL-1Ra), and interleukin-2 (IL-2) in cerebrospinal fluid and MIF in serum differentiate urban children exposed to severe vs. low air pollution. Front. Neurosci. 7:183. doi: 10.3389/fnins.2013.00183

Campbell, B. M., Charych, E., Lee, A. W., and Moller, T. (2014). Kynurenines in CNS disease: regulation by inflammatory cytokines. Front. Neurosci. 8:12. doi: 10.3389/fnins.2014.00012

Denes, A., Pradillo, J. M., Drake, C., Buggey, H., Rothwell, N. J., and Allan, S. M. (2013). Surgical manipulation compromises leukocyte mobilization responses and inflammation after experimental cerebral ischemia in mice. Front. Neurosci. 7:271. doi: $10.3389 /$ fnins.2013.00271
Denes, A., Thornton, P., Rothwell, N. J., and Allan, S. M. (2010). Inflammation and brain injury: acute cerebral ischaemia, peripheral and central inflammation. Brain Behav. Immun. 24, 708-723. doi: 10.1016/j.bbi.2009.09.010

Deretzi, G., Kountouras, J., Polyzos, S. A., Zavos, C., Giartza-Taxidou, E., Gavalas, E., et al. (2011). Gastrointestinal immune system and brain dialogue implicated in neuroinflammatory and neurodegenerative diseases. Curr. Mol. Med. 11, 696-707. doi: 10.2174/156652411797536660

Elenkov, I. J., Wilder, R. L., Chrousos, G. P., and Vizi, E. S. (2000). The sympathetic nerve-an integrative interface between two supersystems: the brain and the immune system. Pharmacol. Rev. 52, 595-638.

Garcia-Bonilla, L., Benakis, C., Moore, J., Iadecola, C., and Anrather, J. (2014). Immune mechanisms in cerebral ischemic tolerance. Front. Neurosci. 8:44. doi: 10.3389/fnins.2014.00044

Geenen, V., Bodart, G., Henry, S., Michaux, H., Dardenne, O., Charlet-Renard, C., et al. (2013). Programming of neuroendocrine self in the thymus and its defect in the development of neuroendocrine autoimmunity. Front. Neurosci. 7:187. doi: 10.3389/fnins.2013.00187

Goyal, D. K., and Miyan, J. A. (2014). Neuro-immune abnormalities in autism and their relationship with the environment: a variable insult model for autism. Front. Endocrinol. (Lausanne) 5:29. doi: 10.3389/fendo.2014.00029

Heneka, M. T., Kummer, M. P., and Latz, E. (2014). Innate immune activation in neurodegenerative disease. Nat. Rev. Immunol. 14, 463-477. doi: 10.1038/nri3705

Iadecola, C., and Anrather, J. (2011). The immunology of stroke: from mechanisms to translation. Nat. Med. 17, 796-808. doi: 10.1038/nm.2399

Kamimura, D., Yamada, M., Harada, M., Sabharwal, L., Meng, J., Bando, H., et al. (2013). The gateway theory: bridging neural and immune interactions in the CNS. Front. Neurosci. 7:204. doi: 10.3389/fnins.2013.00204

Moreno-Smith, M., Lutgendorf, S. K., and Sood, A. K. (2010). Impact of stress on cancer metastasis. Future Oncol. 6, 1863-1881. doi: 10.2217/fon.10.142

Onore, C. E., Careaga, M., Babineau, B. A., Schwartzer, J. J., Berman, R. F., and Ashwood, P. (2013). Inflammatory macrophage phenotype in BTBR $\mathrm{T}+\mathrm{tf} / \mathrm{J}$ mice. Front. Neurosci. 7:158. doi: 10.3389/fnins.2013.00158

Rivest, S. (2009). Regulation of innate immune responses in the brain. Nat. Rev. Immunol. 9, 429-439. doi: 10.1038/nri2565

Theoharides, T. C., Asadi, S., and Patel, A. B. (2013). Focal brain inflammation and autism. J. Neuroinflammation 10:46. doi: 10.1186/1742-2094-10-46

Wraith, D. C., and Nicholson, L. B. (2012). The adaptive immune system in diseases of the central nervous system. J. Clin. Invest. 122, 1172-1179. doi: 10.1172/JCI58648

Conflict of Interest Statement: The authors declare that the research was conducted in the absence of any commercial or financial relationships that could be construed as a potential conflict of interest.

Received: 29 October 2014; accepted: 09 November 2014; published online: 03 December 2014

Citation: Denes A and Miyan JA (2014) Brain-immune interactions in health and disease. Front. Neurosci. 8:382. doi: 10.3389/fnins.2014.00382

This article was submitted to Neuroendocrine Science, a section of the journal Frontiers in Neuroscience.

Copyright (C) 2014 Denes and Miyan. This is an open-access article distributed under the terms of the Creative Commons Attribution License (CC BY). The use, distribution or reproduction in other forums is permitted, provided the original author(s) or licensor are credited and that the original publication in this journal is cited, in accordance with accepted academic practice. No use, distribution or reproduction is permitted which does not comply with these terms. 